\title{
El impacto del turismo en la economía local. Una necesidad de medir en el Ecuador
}

\section{The impact of tourism on the local economy. A need to measure in Ecuador}

\author{
Yonimiler Castillo Ortega ${ }^{1 *}$, Margarita Andrea Polo Espinoza ${ }^{1}$ y Fernando David Uruchima Juca ${ }^{1}$ \\ ${ }^{1}$ Universidad Católica de Cuenca \\ *ycastilloo@ucacue.edu.ec
}

DOI: https://doi.org/10.26871/killkana_social.v2i3.344

\begin{abstract}
Resumen
El presente trabajo de investigación estudia cómo se mide el impacto de la actividad turística en la economía a nivel local en el Ecuador. La investigación es de carácter exploratoria, se empleó el método de análisis y síntesis que permitió la comprensión de teorías sobre desarrollo económico local y el turismo, así como el método empírico para la comprensión del fenómeno en la realidad del contexto ecuatoriano. Para la recolección de información se aplicó una entrevista a técnicos de turismo de 20 territorios y a 12 expertos que tienen experiencia en el desarrollo turístico de todo el país. Como resultados se obtuvo que el $70 \%$ de los territorios conoce el impacto de la actividad turística en el desarrollo económico local. Así mismo, es medido por el $60 \%$ de ellos. Los instrumentos más utilizados por estas localidades son las encuestas y los indicadores turísticos. De estos últimos, el gasto por turista y la cantidad de turistas fueron los indicadores más utilizados y conocidos por las localidades y expertos. Las localidades más avanzadas en esta medición en el país son Galápagos y Quito ya que fueron considerados por el mayor porcentaje de entrevistados como territorios de vocación turística que emplean modelos para cumplir con la función en estudio. Sin embargo la región Amazónica es aquella en la que medir el impacto del turismo a nivel local se considera algo importante, pero es un proceso que no se lleva a cabo. Es así que se constata la necesidad de un modelo, metodología o instrumento que permita medir el impacto de la actividad turística en la economía local, como herramienta para la toma de decisiones en el ámbito turístico a nivel local en Ecuador.
\end{abstract}

Palabras clave: Desarrollo Económico Local, Desarrollo Económico Local, Turismo.

\begin{abstract}
This research work studies how the impact of tourism activity on the local economy in Ecuador is measured. The research is exploratory in nature, using the method of analysis and synthesis that allowed the understanding of theories on local economic development and tourism, as well as the empirical method for understanding the phenomenon in the reality of the Ecuadorian context. For the collection of information an interview was applied to tourism technicians from 20 territories and 12 experts who have experience in tourism development throughout the country. As a result, it was found that $70 \%$ of the territories know the impact of tourism activity on local economic development. Likewise, it is measured by $60 \%$ of them. The instruments most used by these locations are surveys and tourism indicators. Of the latter, the expense per tourist and the number of tourists were the indicators most used and known by localities and experts. The most advanced localities in this measurement in the country are Galápagos and Quito since they were considered by the highest percentage of interviewees as territories of tourist vocation that use models to fulfill the function under study. However, the Amazon region is one in which measuring the impact of tourism at the local level is considered important, but it is a process that is not carried out. Thus, the need for a model, methodology or instrument to measure the impact of tourism activity on the local economy, as a tool for decision making in the field of tourism at the local level in Ecuador is confirmed.
\end{abstract}

Key words: Local Economic Development, Impact, Tourism.

\section{Introducción}

El desarrollo local juega un papel importante en los diferentes territorios, debido al aprovechamiento de los recursos y potencialidades endógenas, esto con el objetivo de promover el dinamismo económico y mejorar la calidad de vida de la población local, sobre todo para los países subdesarrollados y que presentan severas crisis económicas. Las teorías sobre el desarrollo local surgen por la necesidad de dar una solución a las diferencias territoriales, la pobreza $\mathrm{y}$ al neoliberalismo. Citando a Buarque planteo que el 
desarrollo local "es un proceso endógeno registrado en pequeñas unidades territoriales y asentamientos humanos capaz de promover el dinamismo económico y la mejoría en la calidad de vida de la población." (Varisco, 2008, p.34) Por eso las localidades mediante el uso de estrategias deben aprovechar al máximo posible las capacidades de los diferentes actores económicos, sociales, políticos, ambientales, etc. En la parte económica se da un proceso de crecimiento y cambio estructural de la economía de una localidad con sus diversas actividades, con el fin de mejorar condiciones de crecimiento económico.

\section{Figura 1. Peso del consumo turístico receptor en el PIB}

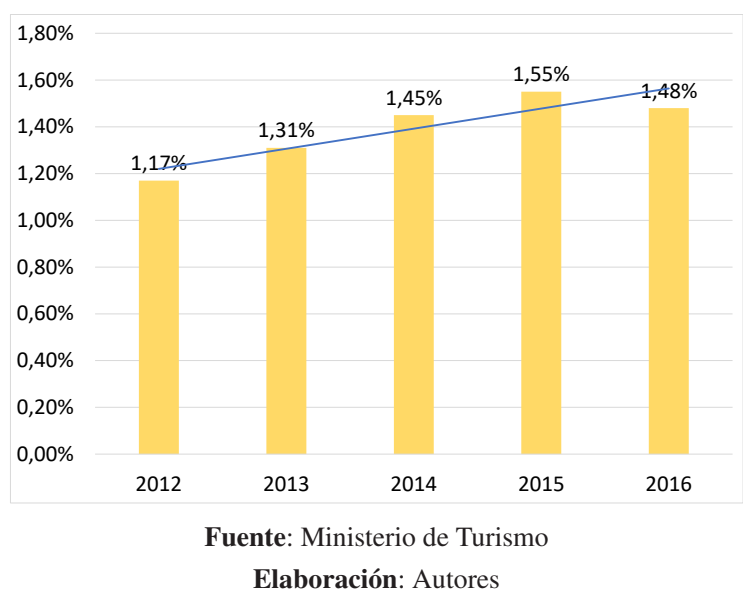
para su desarrollo local es el turismo, citando a Santos "ve al turismo como la gran salvación de mundo rural en declive" (Bringas, Nora, González, y Israel, 2004, p.7). Sancho (2004), menciona que "al turismo se le considera como un sector generador de oportunidades, capaz de crear negocios y empleo.”(p. 1), siendo una actividad económica que ha contribuido a la generación de empleo e ingresos en las diversas comunidades que han visto de la actividad turística una fuente de riqueza. Esto se ha podido percibir en países europeos en donde su principal fuente de ingreso es el turismo, así como en diversas localidades de Latinoamérica y el Caribe, por lo que es indispensable conocer su impacto económico a través de instrumentos, herramientas, metodologías, etc., que en la actualidad existen modelos para medir a nivel agregado de la economía como es el modelo de matriz de contabilidad social, matriz de doble entrada, los modelos econométricos como el Input-Output, la Cuenta Satélite de Turismo que es utilizada por la Organización Mundial de Turismo, etc. Sin embargo a nivel local todavía existen muchas falencias para su medición.

En la presente investigación estudia este fenómeno en el contexto del Ecuador donde existen muchas falencias al momento de medir el impacto del turismo en la economía aun cuando el turismo se ha incrementado en los últimos años. Según datos del Ministerio de Turismo desde el año 2012 al 2016, la entrada de extranjeros al país ha incrementado en una tasa promedio de $3,10 \%$. De igual modo se han incrementado los ingresos por turismo con una tasa promedio de 9,27\%. La participación del turismo en el PIB ha experimentado un crecimiento favorablemente para Ecuador con una tasa promedio de 6,26\%, siendo importante para el desarrollo.
Figura 2. Entrada de extranjeros al Ecuador

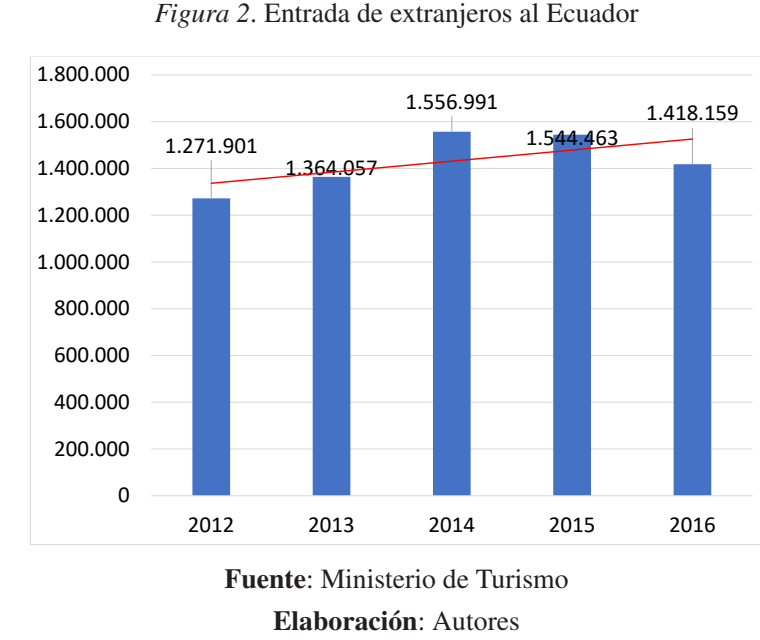

Figura 3. Balanza Turística Ingresos

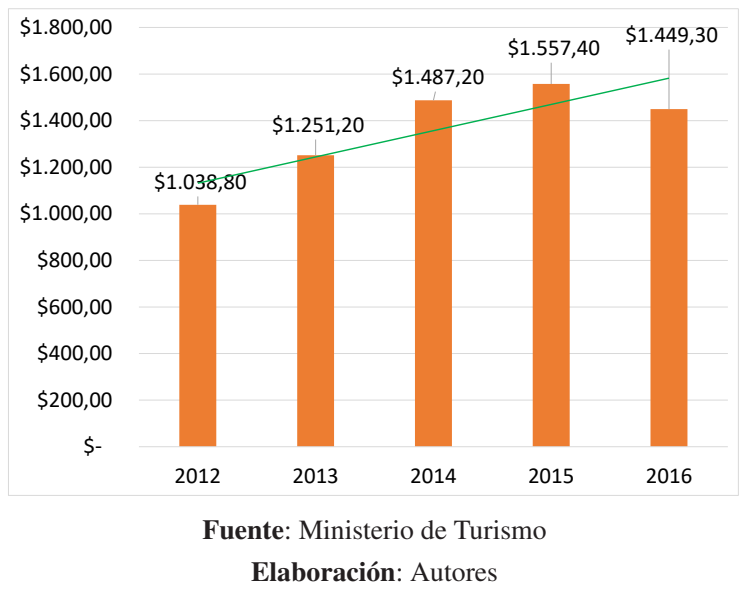

Por lo tanto la presente investigación tiene como objetivo analizar las formas de medir el impacto de la actividad turística en la economía local en Ecuador y defiende la idea que la medición del turismo es una necesidad ser medida en Ecuador y debe haber algún diseño o instrumentos 
que estudie este impacto para la toma de decisiones. Para cumplir con la investigación se utilizó el método teórico, el método empírico, además de técnicas como la revisión bibliográfica, las entrevistas estructuradas y semiestructuradas, siendo una investigación con un alcance exploratorio.

\section{Marco teórico}

El presente acápite tiene como objetivo analizar las principales tendencias teóricas y empíricas sobre la medición del impacto del turismo a escala local. Para esto se iniciará con una revisión de algunos antecedentes sobre el desarrollo local que servirán como base para la comprensión del desarrollo turístico a este nivel.

\subsection{Antecedentes teóricos del Desarrollo local}

A partir de la Segunda Guerra Mundial, con el fin de acelerar el crecimiento y traer consigo el progreso de los países pobres, nace el concepto de desarrollo. El mismo que busca todas las existencias para el bienestar humano, en los aspectos económicos, sociales, políticos, culturales y del medio ambiente, que implique cambios en las actitudes y conductas individuales y grupales que impactan las estructuras que tiene cada institución.

Una vez generada dicha definición, surge el desarrollo local, sobre el cual Alburquerque alegó que el desarrollo pasó de concebirse como un proceso a nivel macroeconómico, es decir, nacional a un proceso territorial, viendo la necesidad de estudiar recursos endógenos y centrados en un territorio específico. (Díaz, 2017)

De igual manera, el autor (Gallicchio, 2003), desde su punto de vista, afirma que el desarrollo debe actuar de forma local debido a los cambios experimentados por la globalización. Dicho esto, define al desarrollo local como un proceso de crecimiento económico y cambio estructural que mejora el nivel de bienestar de una localidad o región, y que tiene por objeto promover el dinamismo económico de la población. Por lo tanto, cuando una comunidad experimenta un proceso de cambio estructural, se está ante un desarrollo local endógeno, que según Coraggio, tiene cuatro componentes: económico, social, cultural y político. (Gonzáles Flores, 2008, p.3)

Citando a Alburquerque define al desarrollo económico local como un "proceso de desarrollo participativo que fomenta los acuerdos de colaboración entre los principales actores públicos y privados de un territorio, posibilitando el diseño y la puesta en práctica de una estrategia de desarrollo territorial común." (Alfaro y Costamagna, 2015, p.46) Dicha estrategia debe cumplir con el objetivo de crear nuevas oportunidades de empleo e ingreso para la sociedad a nivel local. Todo esto basándose en los recursos existentes en cada localidad.

El desarrollo económico local es un proceso de crecimiento y cambio estructural de la economía de una ciudad, provincia o región con el fin de mejorar condiciones de crecimiento económico, caracterizada por un sistema de producción que permite a los empresarios y autoridades usar eficientemente los factores productivos, generar economías de escala e incrementar la productividad a niveles que permiten mejorar la competitividad en los mercados, además se pretende mejorar la calidad de vida de la población y la generación de empleo.

Como parte de las alternativas de desarrollo local, muchos territorios han incursionado en la actividad turística para reactivar la economía de su localidad. En seguida se abordarán algunos aspectos teóricos del turismo y su impacto en el desarrollo económico local, así como los diferentes modelos que se han utilizado para medirlo.

\subsection{El Turismo y su impacto en el desarrollo económico local}

Como se mencionó anteriormente, el turismo es una actividad propulsora de nuevas oportunidades, especialmente "para zonas subdesarrolladas y periféricas, que se ha convertido en el motor de las mismas para el alcance de metas tanto económicas como sociales." (Narváez, Mercy; Fernández, 2013, p. 129) Es debido a esto, que el actual trabajo de investigación hará énfasis en el turismo y el desarrollo local.

Para determinar el impacto del turismo en el desarrollo económico, se aplican una serie de instrumentos, herramientas, metodologías o modelos de medición. Algunos de ellos, caracterizados fundamentalmente por medir el impacto de la actividad turística a nivel agregado de la economía, son los siguientes:

La OMT ${ }^{1}$ plantea que "en el análisis económico del turismo, se debe distinguir entre la 'contribución económica' del turismo que se refiere al efecto directo del turismo y que se puede medir mediante la $\mathrm{CST}^{2}$, y el 'impacto económico' del turismo de, que es un concepto mucho más amplio que encapsula los efectos directos, indirectos e inducidos del turismo y que debe ser estimado mediante la aplicación de modelos".

Así mismo, de acuerdo con (García Mestanza y Caballero Galeote, 2015) existen varios modelos que siguen este propósito, y se clasifican en cuantitativos y cualitativos.

Dentro de los modelos económicos de medición cuantitativos, se encuentran los econométricos que establecen la relación entre una o más variables. Este modelo usa las regresiones para llevar a cabo modelos macroeconómicos de medición regional. Uno de los más utilizados es el denominado Input-Output.

El modelo de matriz de contabilidad social, consiste en "la representación de las relaciones económicas más importantes entre los agentes de una economía en un momento del tiempo" (García Mestanza y Caballero Galeote, 2015, p.11). Y según Argüelles \& Benavides, (2003) “en comparación con el Input-Output, ofrece una mayor cantidad de información, captando con mayor precisión los efectos de cambios exógenos." (p. 165) Cuando se trata de medir el impacto económico del turismo, este es uno de

\footnotetext{
${ }^{1}$ Organización Mundial del Turismo

${ }^{2}$ Cuenta Satélite del Turismo
} 
los instrumentos más precisos pues se puede dar cabida a la segregación de datos que en si brinda un resultado más acertado.

Como se conoce, la investigación cualitativa trabaja con características, cualidades, sentimientos, experiencias etc. Un modelo de medición cualitativo se realiza a través de una matriz de doble entrada en la que se reflejen opiniones expresadas por distintos grupos de interés. Este modelo tiene la finalidad de conocer diferentes críticas de la incidencia o impacto de un fenómeno. La información es recolectada mediantes entrevistas.

Sin embargo a nivel local todavía existen muchas falencias. De acuerdo con Figuerola Palomo, Fernández Muñoz, Marzal Baro, Gómez Bruna, \& Martín Duque, (2015), quien realizó un proyecto bajo esta temática en España, "los indicadores de desarrollo turístico con proyección local sirven para medir las influencias de las llegadas o crecimiento turístico en un determinado destino". (p. 48)

El estudio de Figuerola (2015), se ha centrado en los indicadores de naturaleza económica como principal medio para la medición de la incidencia del turismo a nivel local puesto que estos se han convertido en la base para la elaboración de modelos o instrumentos de acción que más tarde permitirían plantear estimaciones en el ámbito turístico.

En seguida se detalla un cuadro en el que se exponen algunos indicadores y la función que cumple cada uno:

Tabla 1 Indicadores de naturaleza económica para medir el impacto del turismo a nivel local

\begin{tabular}{|c|c|}
\hline Indicador & Función que cumple \\
\hline $\begin{array}{l}\text { Nivel de producción turística direc- } \\
\text { ta (PTD) }\end{array}$ & $\begin{array}{l}\text { Mide la evolución de las ventas que las } \\
\text { empresas y sectores, directamente rela- } \\
\text { cionadas y relacionados con el gasto de } \\
\text { los turistas realizan. }\end{array}$ \\
\hline $\begin{array}{l}\text { Renta Turística y su relación con } \\
\text { el PIB local o regional. Según pe- } \\
\text { ríodos anuales o trimestrales y su } \\
\text { comparación temporal (RTRL) }\end{array}$ & $\begin{array}{l}\text { Valora la parte de la producción turísti- } \\
\text { ca que se transmite como renta o valo- } \\
\text { res añadidos al Producto Interior Bruto } \\
\text { del país. Es un indicador básico, puesto } \\
\text { que resume el conjunto de la aportación } \\
\text { económica del turismo en el marco del } \\
\text { área geográfica que se desee conocer y } \\
\text { proyectar políticamente. }\end{array}$ \\
\hline $\begin{array}{l}\text { Indicador de competitividad de la } \\
\text { demanda turística }\end{array}$ & $\begin{array}{l}\text { Establece por medio de un indicador cuál } \\
\text { es el grado o escala de competitividad de } \\
\text { los diferentes municipios, permitiendo } \\
\text { una comparación homogénea }\end{array}$ \\
\hline $\begin{array}{l}\text { Multiplicador de la producción tu- } \\
\text { rística sobre el resto del sistema } \\
\text { económico }\end{array}$ & $\begin{array}{l}\text { Permite valorar los efectos indirectos e } \\
\text { inducidos causados por el gasto de los } \\
\text { turistas, de manera que puede tenerse } \\
\text { una percepción global de los efectos del } \\
\text { turismo }\end{array}$ \\
\hline $\begin{array}{l}\text { Valoración del esfuerzo presupues- } \\
\text { tario municipal en la promoción de } \\
\text { la demanda turística }\end{array}$ & $\begin{array}{l}\text { La comparación de la acción presupues- } \\
\text { taria municipal, y la valoración conse- } \\
\text { guida de los efectos e influencia del tu- } \\
\text { rismo, medirá la eficiencia de la acción } \\
\text { política, respecto al presupuesto determi- } \\
\text { nando la validez de la cantidad, así como } \\
\text { la distribución de las partidas. }\end{array}$ \\
\hline $\begin{array}{l}\text { Correlación anual o dependencia } \\
\text { del turismo en el destino, respecto } \\
\text { al conjunto del turismo en el país } \\
\text { (residentes y no residentes) }\end{array}$ & $\begin{array}{l}\text { El indicador establece en un cuadro com- } \\
\text { parativo, como los comportamientos de } \\
\text { la estrategia turística ha tenido lugar a } \\
\text { nivel del municipio, y nivel nacional, } \\
\text { manifestando el grado de dependencia } \\
\text { posible, entre el conjunto total, y cada } \\
\text { una de sus partes }\end{array}$ \\
\hline
\end{tabular}

Tabla 1 Indicadores de naturaleza económica para medir el impacto del turismo a nivel local...(Continuación)

\begin{tabular}{|l|l|}
\hline \multicolumn{1}{|c|}{ Indicador } & \multicolumn{1}{c|}{ Función que cumple } \\
\hline $\begin{array}{l}\text { Indicador de dependencia del creci- } \\
\text { miento en los destinos turísticos en } \\
\text { función de la variación de la renta } \\
\text { de los países emisores }\end{array}$ & $\begin{array}{l}\text { Es conveniente disponer de un instru- } \\
\text { mento de previsión, que permita indicar } \\
\text { o alertar, como los comportamientos de } \\
\text { las demandas turísticas generales, pue- } \\
\text { den influir en el marco local. }\end{array}$ \\
\hline $\begin{array}{l}\text { Valoración en el ámbito municipal } \\
\text { de indicadores de productividad de } \\
\text { empleo e inversión hotelera en los } \\
\text { destinos turísticos }\end{array}$ & $\begin{array}{l}\text { La eficacia alcanzada por los factores } \\
\text { productivos es una magnitud necesaria si } \\
\text { se pretende mejorar en cuanto a los resul- } \\
\text { tados parciales y generales del proceso } \\
\text { de producción }\end{array}$ \\
\hline
\end{tabular}

Fuente: (Figuerola Palomo y cols., 2015)

Las anteriores, son algunas de las formas de medir el impacto del turismo a nivel nacional y local. Sin embargo también se han dado algunas experiencias en donde se ha medido dicho impacto de distintas maneras. Por ejemplo en Guatemala, para determinar esta incidencia, el Municipio de San Francisco Zapotitlán realizó cálculos en base a estimaciones de gastos de hospedaje, recreación y alimentación, así como otros gastos promedio por visitante. De igual manera, se analizó el indicador de pobreza utilizando un enfoque metodológico estadístico que compara el cambio en el nivel de pobreza entre 1998 y 2006. A partir de esto, se obtuvo como resultado que el turismo sostiene la mayor reducción de este nivel en San Francisco Zapotitlán, aportando al desarrollo local y trayendo consigo aspectos positivos tales como: el aumento del nivel educativo, fomento de empresas y conservación ambiental. También se pudo determinar que a nivel local el turismo tiene mayor importancia que a nivel nacional, representando $8 \%$ del PIB del departamento de Retalhuleu y $78 \%$ del empleo formal en el municipio de San Francisco Zapotitlán. (Díaz, 2017)

Por otra parte, en Zacatecas-México, luego de haberse declarado al centro histórico de la ciudad como Patrimonio Cultural de la Humanidad se realizó una evaluación del impacto económico del turismo en la misma. Se examinó el cambio registrado en el giro de las actividades económicas en la ciudad antes y después de la declaración así como la variación de indicadores como por ejemplo la apertura de nuevos servicios turísticos, cantidad de turistas, etc. Una vez realizado el análisis con base en la elaboración de cuadros, gráficos y cartografía en varias escalas se pudo determinar que no se alcanzaron "los resultados esperados ya que su derrama económica no ha sido importante, en cambio la terciarización de su economía ha seguido la tendencia natural del país y no la de la generación de empleos relacionados con el turismo" (Sicilia Muñoz, 2012)

Esta experiencia como otras, utiliza algunas herramientas. Sin embargo todavía no se ha podido encontrar un modelo o metodología que permita medir el impacto turístico a nivel local. Lo cual se constata también en el presente estudio en el Ecuador: la Facultad de Ciencias Políticas y Administrativas de la Universidad Nacional de Chimborazo realizó un análisis del impacto del turismo 
en el crecimiento económico del Ecuador, período 2000 2014. Este estudio se llevó a cabo mediante la implementación de un modelo de regresión lineal estimado por el método de mínimos cuadrados ordinarios. Los resultados de esa estimación mostraron que existe una relación directa entre el sector turístico y la tasa de crecimiento del Producto Interno Bruto (PIB) real en estos años. Además el modelo utilizado trajo resultados que evidencian que "el turismo impacta positivamente en el crecimiento económico del país, en un $0.27 \%$ por cada $\%$ adicional en que varié los ingresos turísticos y en $0.17 \%$ de la fuerza laboral." (Caguana Andino y Janeta Aulla, 2016)

En base a las experiencias descritas se observó que en cada uno de los casos se llevan a formas diferentes de medir el impacto del turismo en el desarrollo local. En el Ecuador se aplica un modelo econométrico, sin embargo este análisis se desarrolló a nivel nacional. En Guatemala y México se llevaron a cabo mediciones en localidades pero estas se realizaron a través de instrumentos y herramientas que no constituyen un modelo específico.

Por lo tanto, a nivel internacional se comprueba una falencia metodológica y en el Ecuador también se corrobora la inexistencia de un modelo. Demostrándose así la necesidad de diseñar un instrumento que lleve a cabo este proceso a nivel local.

\section{Metodología}

La presente es una investigación de tipo mixta pues se realiza desde los enfoques cuantitativos y cualitativos. El primero se utilizó para medir la cantidad de territorios que utilizan modelos $\mathrm{u}$ otros instrumentos para evaluar el impacto del turismo en la economía local, así como la obtención de los indicadores que utilizan. El segundo para encontrar las principales características que identifican las formas de medir el impacto del turismo en la economía en el Caso de Ecuador. El alcance es exploratorio, ya que pretende la demostración de una problemática en el ámbito turístico a nivel local en el país.

Para el desarrollo de la presente investigación se utilizaron métodos teóricos tales como el método histórico con el que se analizaron las tendencias y la evolución del turismo. Así mismo se aplicó el procedimiento de análisis y síntesis para la elaboración del marco teórico, que permitió el estudio de teorías sobre desarrollo económico local y el turismo.

La técnica utilizada para la recolección de dicha información fue la revisión bibliográfica de artículos y publicaciones a través de la base de datos científica.

Por otra parte, el método empírico de la encuesta se empleó para la comprensión del fenómeno turístico a nivel local y su forma de medición en la realidad del contexto ecuatoriano.

Se usaron técnicas para la obtención de información primaria. Entre estas están entrevistas estructuradas aplicadas a directores, técnicos y analistas de turismo de GADs Municipales en 20 localidades ecuatorianas. Así mismo se aplicaron entrevistas semi estructuradas a 12 expertos en turismo del país los mismos que son docentes universitarios, directores académicos, y técnicos del MINTUR que permitieron validar la información obtenida de los territorios.

\section{Resultados}

En este acápite se pretende demostrar que, en el caso del Ecuador, se mantiene la insuficiencia de herramientas y modelos para evaluar la capacidad de la actividad turística para generar relaciones económicas locales. Para esto se aplicó una entrevista estructurada a técnicos de turismo de los 20 territorios más importantes en cuanto a turismo local en Ecuador. La validez de estos resultados se comprobó que es buena, a partir del Alpha de Cronbach que resultó ser 0,790 para la medición del impacto del turismo en la economía local y 0,897 para la necesidad de diseñar un modelo o instrumento que mida este fenómeno, así como su implementación.

Además se realizaron 12 entrevistas a expertos que conocen de la actividad turística en el Ecuador, entre ellos funcionarios del Ministerio de Turismo de Ecuador, Dirigentes de las Cámaras y Fundaciones de Turismo, así como a Profesores Universitarios. El Alpha de Cronbach en este caso arrojó el valor de 0,897 , lo que verifica la validez de las respuestas de los expertos.

En primera instancia, y en relación a la información que manejan las localidades con respecto al objeto de estudio de esta investigación se pudo determinar que el $60 \%$ de localidades cuenta siempre con información periódica sobre la actividad turística de su territorio, en base a la cual toman decisiones para el desarrollo. El $10 \%$ de entrevistados que nunca cuenta con este tipo de información son las ciudades de Sigsig y Guaranda, las mismas que se basan en proyectos, información informal y en planes de trabajo del Alcalde, para la toma de decisiones en el ámbito turístico.

El $85 \%$ de localidades entrevistadas dispone de mecanismos para captar el comportamiento de la actividad turística. Entre los mecanismos más utilizados por dichos territorios se encuentran las encuestas y estadísticas proporcionadas por diferentes instituciones como por ejemplo del ITUR y MINTUR.

Contar con este tipo de información es un aspecto relevante para conocer la importancia de la actividad turística en las economías locales, así como para facilitar la toma de decisiones de las autoridades. Es por esto que el $60 \%$ de territorios afirma utilizar siempre la información disponible para dichos fines.

De igual manera, según expertos de la Universidad Espíritu Santo de Guayaquil, los criterios e información en base a los cuales se toman decisiones para impulsar el desarrollo turístico en territorios ecuatorianos consisten generalmente en las tendencias del turismo actual, estrategias que responden a intereses políticos, indicadores, y una vez más se considera a las estadísticas para este fin. 
Medir el impacto del turismo en el desarrollo local se ha convertido en un verdadero reto tanto nacional como internacionalmente. A pesar de que el $70 \%$ de directores y técnicos en turismo afirma que en su localidad sí conocen el impacto de la actividad turística en el desarrollo económico de su territorio, y que el $60 \%$ de localidades alegue medirlo; se pudo constatar la falencia metodológica que existe en el país, puesto que, como se muestra en la figura $\mathrm{N}^{\circ} 4$., los instrumentos más utilizados por los entrevistados para medir el impacto son las encuestas e indicadores turísticos.

Figura 4. Instrumentos utilizados en las localidades para medir el impacto de la actividad turística en el desarrollo económico del territorio

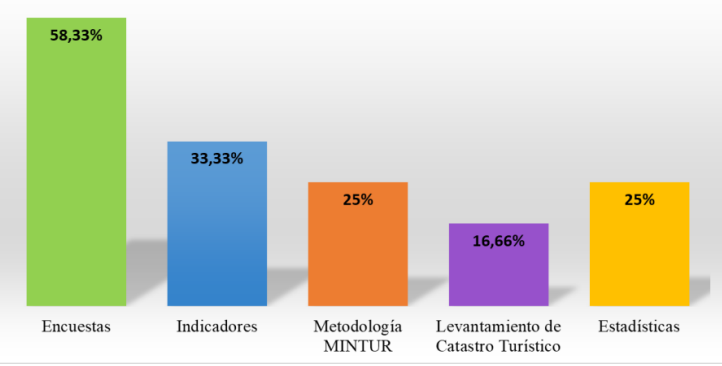

Fuente: Información de campo

Sin embargo en Galápagos, (considerado por el $50 \%$ de expertos en el país como un territorio de vocación turística que utiliza instrumentos, metodologías y otros para medir la incidencia del turismo a nivel local) se pudo notar lo contrario a la afirmación previamente establecida. De acuerdo con una analista de turismo del GAD Municipal de Santa Cruz, dicho procedimiento se lleva a cabo a través de un modelo denominado SLOT. El modelo SLOT permite, en base a información y estadísticas, identificar gráficamente como está el desarrollo del turismo. Este sistema tiende a ser circular, por lo que se puede observar cuando un indicador turístico está distorsionando el modelo, sobresaliendo de la circunferencia. De este modo se puede ir modelando el sistema en base a aquellos indicadores que presentan problemas.

Otro aspecto a destacarse, es el hecho de que en la Amazonía, la medición del impacto del turismo a nivel local, es un proceso poco desarrollado. Todavía existe un $40 \%$ de localidades ecuatorianas que no miden el impacto en estudio, y este porcentaje está conformado por Tena, Puyo, entre otros. En concordancia con esto, el Técnico del área de Turismo y Coordinador encargado de dicho departamento del GAD Municipal de la ciudad de Tena comentó que "en la Amazonia no hay mediciones" y que "saben que es algo importante pero no hay procesos de medición".

Adicional a esto, ciudades como Zaruma y Macas demostraron un escaso conocimiento en estos temas en base a las respuestas brindadas. Sin mencionar que varios de ellos no conocían la información el momento de ser entrevistados, sino que alegaban tener que revisarlo en otros medios y lugares.

Como se mencionó anteriormente, los indicadores turísticos son uno de los medios más utilizados por los entrevistados para medir el impacto de la actividad turística en el desarrollo económico local. No obstante, la mitad de ellos, es decir el $50 \%$, no tienen definido indicadores para este fin. Esto se debe a que esta información es proporcionada por otras entidades. Como por ejemplo en Baños de Agua Santa trabajan en conjunto con el Consejo Provincial, los mismos que a final de cada año les transmiten dicha información. Así mismo en Guaranda trabajan con información proporcionada por el Ministerio de Turismo y están a la espera de su nueva metodología para aplicarla.

Los indicadores más utilizados en las localidades ecuatorianas son la cantidad de turistas, y el gasto por turista. De igual manera, los expertos entrevistados que están enterados de la existencia de estos indicadores, coinciden con estas respuestas. Sin embargo algunos otros mencionados fueron la cantidad de días de visita, las actividades de motivación, etc.

Figura 5. Indicadores definidos en las localidades para medir el impacto de la actividad turística en el desarrollo económico del territorio

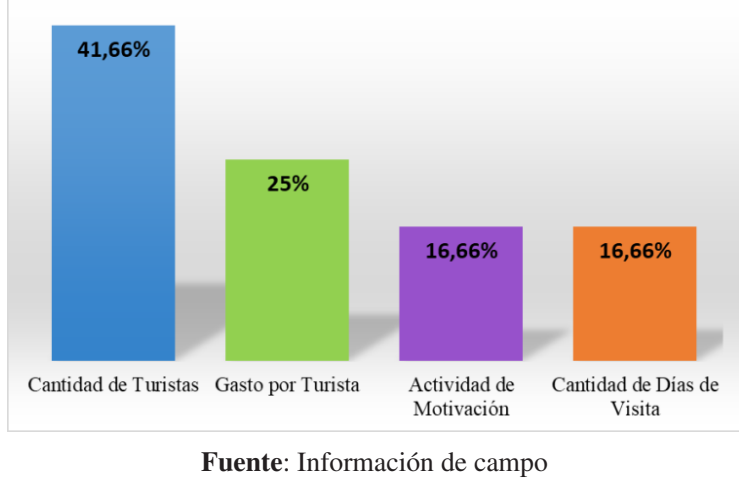

Son pocos los estudios en el Ecuador que miden el impacto de la actividad turística en el desarrollo económico local. En relación a esto, el Director de la Escuela de Turismo y Hotelería de la Pontificia Universidad Católica del Ecuador comentó que "Los estudios hacen generalmente un análisis a nivel regional o nacional. Los datos de información local son menos frecuentes, precisamente por la falta de los mismos." Evidenciando dicha afirmación, el $25 \%$ de expertos alegan desconocer la existencia de este tipo de estudios en el país.

Por otra parte, el porcentaje restante considera en su mayoría estudios como los proyectos que realiza el MINTUR, por ejemplo el "turismo en cifras" y la "Cuenta Satélite". También son mencionados el Observatorio Turístico de Galápagos y Guayaquil, y los artículos y tesis de escuelas de turismo.

Es así que se hace evidente la necesidad de la creación un instrumento, metodología o modelo en el Ecuador que mida el impacto en estudio, ya que como se ha podido 
visualizar, los testimonios y pensamientos detallados de los 20 territorios se complementan y corroboran con las opiniones de 12 expertos en turismo del país. Tanto el $75 \%$ de directores y técnicos, como el 83,33\% de los expertos, consideran a la medición del impacto del turismo en las localidades una actividad indispensable.

Según se muestra en figura $\mathrm{N}^{\circ} 6$., el $95 \%$ de directores y técnicos de las localidades ecuatorianas afirman necesario el diseño de este instrumento.

Figura 6. Porcentaje de localidades que consideran necesario el diseño de una metodología o modelo para medir el impacto de la actividad turística en el desarrollo económico del territorio

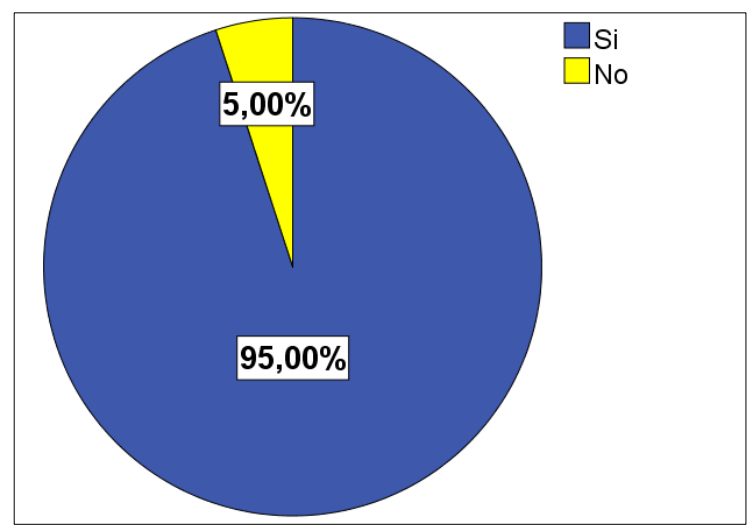

Fuente: Información de campo

Así mismo el $100 \%$ de expertos lo consideran necesario, justificando su afirmación en base a diferentes criterios tales como:

- En función de las cifras que arroje la metodología se tendrá una línea base para la toma de decisiones.

- Generación de estrategias, políticas y lineamientos efectivos para el desarrollo turístico, así como para la planificación.

- Para orientar la inversión turística.

- Erradicar la incongruencia y desactualización de reportes. Y para una rendición de cuentas clara y transparente.

- Para determinar el potencial turístico de cada territorio, y saber qué servicios deben ser mejorados.

- Para saber qué tan bueno es el turismo en el Ecuador, y comprobar el efecto multiplicador del turismo en el país e identificar problemas en la cadena de valor de la experiencia turística a nivel económico.

- Lograr ser más competitivo con sus rivales directos como Colombia, Perú, Costa Rica, etc.
Figura 7. Porcentaje de localidades dispuestas a implementar una metodología o modelo que mida el impacto de la actividad turística en el desarrollo económico del territorio

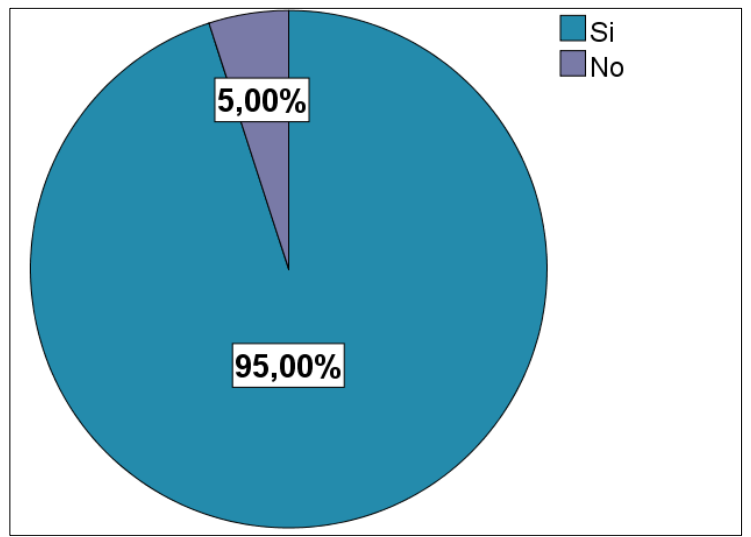

Fuente: Información de campo

En relación a este asunto también se consultó sobre la disposición de implementar un nuevo modelo o instrumento que permita medir el impacto de la actividad turística en el desarrollo económico local. Los resultados son categóricos en este sentido, pues el $95 \%$ de directores y técnicos en turismo manifestaron su disposición de implementarlo en su localidad, la figura $\mathrm{N}^{\circ} 7$. lo representa.

Dicha información se valida con el criterio que emiten los expertos consultados, pues el $100 \%$ de ellos consideran esta implementación muy importante. Especialistas de la ciudad de Guayaquil comentaron que es algo "necesario, urgente, básico" pues beneficiaría tanto a autoridades como a la población en general ya que si se comenzara a implementar un modelo que mida el impacto del turismo en las localidades "los ciudadanos tendrían información necesaria con la que podrían decidir si invertir en este sector o no".

Así mismo, expertos de la Universidad del Azuay confirmaron la relevancia en la implementación de metodologías que permitan medir esos impactos a nivel territorial, puesto que "el turismo siempre genera transformaciones en el territorio, no solo desde el punto de vista económico, sino a partir de factores sociales, ambientales y de percepción en la ciudadanía respecto al impacto del turismo en las comunidades locales."

\section{Conclusiones}

El impacto de la actividad turística a nivel local, es un hecho que se conoce en el $70 \%$ de los territorios. Así mismo, es medido por el $60 \%$ de ellos. Los instrumentos y herramientas más utilizados por estas localidades son las encuestas y los indicadores turísticos.

El $50 \%$ de territorios tienen definido indicadores que los ayuden con esta función, y el 66,66 \% de expertos conocen de ellos. Las respuestas de mayor peso por parte de los dos grupos entrevistados al mencionar algunos indicadores fueron el gasto por turista y la cantidad de turistas.

Se comprobó tanto teóricamente como a través de testimonios de entrevistados la necesidad de una metodología 
o modelo que cumpla con la función en estudio. Las teorías y experiencias nacionales e internacionales demostraron que este es un proceso que se desarrolla en su mayoría a nivel nacional. Las opiniones de técnicos y expertos corroboraron también una falencia metodológica puesto que los instrumentos en mayor porcentaje utilizados no constituyen en sí un modelo o metodología específico.

Los territorios que se han considerado como más avanzados en este aspecto son Galápagos y Quito. En primera instancia porque fueron considerados por el mayor porcentaje de entrevistados como territorios de vocación turística que cuentan con una metodología o modelo para medir la incidencia de la actividad turística en el desarrollo local. La Isla de Santa Cruz de Galápagos fue el único territorio que demostró contar con un modelo que cumpla con dicho fin, además de que su Observatorio de Turismo fue mencionado por muchos como fuente de información turística y elaborador de varios estudios y proyectos en este tema.

Por otro lado, la región Amazónica es aquella en la que la medición del impacto del turismo en el desarrollo económico local se considera como algo importante, pero es un proceso que no se lleva a cabo. Testimonios de territorios como Tena y Puyo corroboraron esta afirmación y otros como Zaruma y Macas demostraron el escaso conocimiento en estos aspectos.

\section{Referencias Bibliográficas}

Alfaro, M. B., y Costamagna, P. (2015). El pensamiento de Francisco Alburquerque en torno al enfoque del Desarrollo Territorial (Vol. 1). doi: 10.1017/ CBO9781107415324.004

Argüelles, Margarita; Benavides, C. (2003). Disponible en: http://www.redalyc.org/articulo.oa?id=63500602. 92-118.

Bringas, R., Nora, L., González, A., y Israel, J. I. (2004). El turismo alternativo : una opción para el desarrollo local en dos comunidades indígenas de Baja California. Economía, Sociedad y Territorio, IV , 551-590.
Caguana Andino, K., y Janeta Aulla, J. (2016). Análisis e Impacto del Turismo en el crecimiento., 123.

Díaz, G. (2017). Turismo y desarrollo local. PASOS. Revista de Turismo y Patrimonio Cultura, 15(2), 333-340.

Figuerola Palomo, M., Fernández Muñoz, J. J., Marzal Baro, F., Gómez Bruna, D., y Martín Duque, C. (2015). Medición del Impacto del Turismo en la Economía, el Empleo y el Medio Ambiente Local.

García Mestanza, J., y Caballero Galeote, L. (2015). MODELOS DE MEDICIÓN DEL IMPACTO TURÍSTICO-ECONÓMICO DE LOS AEROPUERTOS. EL CASO DEL AEROPUERTO DE MÁLAGA-COSTA DEL SOL. , 1-16.

Gonzáles Flores, C. (2008). Desarrollo Local y Turismo.

Sancho, A. (2004). Turismo y desarollo. , 1-13.

Sicilia Muñoz, A. (2012). Evaluación económica del impacto del turismo cultural en la ciudad de Zacatecas. Investigaciones Greográficas, vol. 78(Mx), 89-103.

Varisco, C. (2008). Desarrollo turístico y desarrollo local: La Competitividad de los Destinos Turísticos de Sol y Playa. , 208.

Recibido: 30 de junio de 2018 